\title{
Aberrant expression of MUC4 in ovarian carcinoma: diagnostic significance alone and in combination with MUC1 and MUC16 (CA125)
}

\author{
Subhash C Chauhan ${ }^{1}$, Ajay P Singh ${ }^{1}$, Fernanda Ruiz ${ }^{1}$, Sonny L Johansson ${ }^{2}$, Maneesh Jain ${ }^{1}$, \\ Lynette M Smith ${ }^{3}$, Nicolas Moniaux ${ }^{1}$ and Surinder K Batra ${ }^{1,2,4}$
}

${ }^{1}$ Department of Biochemistry and Molecular Biology, University of Nebraska Medical Center, Omaha, NE, USA; ${ }^{2}$ Department of Pathology and Microbiology, University of Nebraska Medical Center, Omaha, NE, USA; ${ }^{3}$ Department of Preventive and Societal Medicine, University of Nebraska Medical Center, Omaha, NE, USA and ${ }^{4}$ Eppley Institute for Research in Cancer and Allied Diseases, Omaha, NE, USA

\begin{abstract}
Mucins are being implicated in diagnosis, prognosis, and as therapeutic targets due to their aberrant expression in a variety of carcinomas. Here, we have analyzed the expression of MUC4 and have compared its potential usefulness in early detection and prognosis of ovarian carcinoma alone and in combination with other mucin antigens, MUC1 and MUC16. Clinical significance of the differential mucin expression was evaluated by grouping the tumor samples in early (stage I and II) and advanced (stage III and IV) stage cases and histological subtypes (serous, mucinous, endometrioid and clear cell). Correlation of these mucins with patient's survival $(n=63)$ was determined by Kaplan-Meier analysis in order to predict their prognostic value. MUC4 showed significant overexpression in tumor cases $(P<0.0001)$ with highest incidence $(\mathbf{9 2 . 0} \%)$ among all three mucins. A significant overexpression of MUC1 $(P<0.018)$ and MUC16 $(P<0.0001)$ was also observed in 83.0 and $79.0 \%$ of tumor samples, respectively. Notably, MUC4 expression was significantly higher $(P \leq 0.004)$ compared to both MUC1 and MUC16 in early-stage ovarian tumor samples with $100 \%$ incidence. In advanced stage ovarian tumors, all the mucins displayed overall comparable expression, nonetheless, MUC4 had highest prevalence (88.0\%) compared to MUC1 (84.0\%) and MUC16 (81.0\%). A combined panel of MUC4 with MUC16 detected $100 \%$ of the late-stage tumor cases without compromising the specificity. Among histological subtypes, only MUC4 displayed $100 \%(n=5)$ sensitivity in mucinous ovarian tumors, while MUC1 and MUC16 detected 40 and $20 \%$ cases, respectively. The expression of MUC4, however, did not significantly correlate with the survival of the ovarian cancer patient, while a significant correlation of MUC16 with poor prognosis was observed. In conclusion, our study demonstrates that MUC4 could be a potential candidate marker for early diagnosis of epithelial ovarian carcinoma and can be utilized in combination with MUC16 to achieve greater sensitivity for the detection of late-stage tumors.
\end{abstract}

Modern Pathology (2006) 19, 1386-1394. doi:10.1038/modpathol.3800646; published online 28 July 2006

Keywords: Mucins; MUC1; MUC4; MUC16; CA125; ovarian cancer

Ovarian cancer is the fourth leading cause of cancerrelated death in women. According to an estimate by the American Cancer Society, 22220 new cases were diagnosed and 16210 women died due to ovarian cancer in the year 2005. ${ }^{1}$ Epithelial ovarian carcinoma (EOC) is the most common (90\% cases) and malignant form of ovarian cancer and is frequently associated with widespread intraperitoneal metastasis. Most patients present with advanced disease

Correspondence: Dr SK Batra, PhD, Department of Biochemistry and Molecular Biology, Eppley Institute for Research in Cancer and Allied Diseases, University of Nebraska Medical Center, Omaha, NE 68198-5870, USA.

E-mail: sbatra@unmc.edu

Received 3 April 2006; revised and accepted 15 May 2006; published online 28 July 2006 at the time of diagnosis, for which highly effective curative therapy is currently unavailable. ${ }^{2-4}$ Hence, there is an urgent need of ultrasensitive, reliable and effective biomarker(s) for the early detection of this disease in order to minimize the high morbidity and deaths in ovarian cancer patients. Current strategies for the detection of EOC are based on biochemical markers, such as CA125, and biophysical markers assessed by ultrasound and/or Doppler imaging of the ovaries. ${ }^{4,5}$ Unfortunately, the clinical utility of these strategies for the early detection of EOC using these modalities have been limited due to the lack of specificity and predictive value. ${ }^{6-8}$

Owing to the surface epithelial origin of EOC, mucins can be interesting biomolecules to serve as markers of diagnostic significance. ${ }^{9,10}$ To date, 20 human mucins have been identified and categorized 
into two classes (secreted/gel forming and membrane bound) based on their structural characteristics and physiological fates. ${ }^{11,12}$ Mucins are produced by secretory epithelial cells for the lubrication and protection of ducts and lumen within the human body. ${ }^{10,13,14}$ However, mucins are also believed to play an important role in the pathogenesis of benign and malignant diseases of secretory epithelial cells. ${ }^{10,11,15}$ An aberrant expression of mucins has been reported in a variety of carcinomas. CA125, a tumor biomarker being used in clinics for the diagnosis of EOC, is actually a mucin (MUC16). ${ }^{16,17}$ Despite being a sensitive marker, the utility of CA125/MUC16 is limited owing to its elevation in benign conditions such as, endometriosis, pelvic inflammatory disease and pregnancy. ${ }^{2,3,5}$ Another mucin, MUC1 has also been studied in relation to its aberrant expression and pathological functions in ovarian carcinoma. MUC1 is a transmembrane mucin that is overexpressed in ovarian cancer and has been shown to influence the metastatic ability of ovarian carcinoma. ${ }^{18}$ Currently, it is also being explored as a target for antibodymediated tumor therapy. ${ }^{19,20}$

MUC4, a high molecular weight membrane-bound mucin is aberrantly expressed in several types of carcinomas. ${ }^{10,11,14}$ It is a multifunctional protein that is implicated in numerous cellular functions including cell adhesion, motility, signal transduction, tissue regeneration and differentiation, and tumor growth and metastasis. ${ }^{10,11,14,21}$ An earlier study has shown that MUC4 is aberrantly expressed in ovarian carcinoma at transcript level and a significant decrease in MUC4 expression is reported with disease progression. ${ }^{22}$ However, as tumor is a heterogeneous mass of tissue and genes can be regulated post-transcriptionally, it is imperative to study the expression of a gene at protein level in order to predict its association with clinical outcome. In this study, we have investigated the expression of MUC4 in ovarian tumors and normal ovarian tissues using immunohistochemical (IHC) analysis. Expression profiles of MUC1 and MUC16 have also been studied in the same samples to compare the clinical significance of MUC4 alone and in combination with these mucins. Our studies clearly demonstrate that MUC4 can be a useful marker for early diagnosis of ovarian carcinoma and can be used in combination with MUC16/ CA125 to achieve better sensitivity. In addition, we show that the expression of MUC16 correlates with a poor prognosis, while the expression of MUC4 indicates a trend toward the short survival of the ovarian cancer patients.

\section{Materials and methods}

\section{Tissue Specimens}

In total, 63 ovarian cancer (15 frozen and 48 paraffin embedded) and 10 normal ovarian (six frozen and four paraffin embedded) tissue samples were used in this study. Samples were obtained after approval of the protocol by the Institutional Review Board (IRB) at the University of Nebraska Medical Center, Omaha, NE, USA. Thick paraffin sections $(4 \mu \mathrm{m})$ were stained with hematoxylin and eosin for pathological evaluation. The histological type (according to WHO 2003) and pathological stage (according to AJCC) were assessed by a surgical pathologist (SLJ).

\section{Immunohistochemistry}

Frozen tissues were cut into small pieces and embedded in OCT compound (Sakura Fine technical Co., Tokyo, Japan) for cryosectioning. Sections $(4 \mu \mathrm{m})$ were cut on a LEICA CM 1850 cryostat and mounted on super-frost, positively charged glass slides. Sections were fixed in chilled methanol and kept at $-20^{\circ} \mathrm{C}$ for at least $10 \mathrm{~min}$. For paraffin-embedded blocks, sections $(4 \mu \mathrm{m})$ were cut by tissue microtome and mounted on the slides. Sections were deparaffinized using EZ-DeWax $^{\mathrm{TM}}$ (Bio Genex, San Ramon, CA, USA) and then rehydrated with graded alcohols. Heat-induced antigen retrieval was performed in citrate buffer ( $\mathrm{pH}$ 6.0) by heating slides in a microwave oven at $700 \mathrm{~W}$ for $15 \mathrm{~min}$ and the sections were processed for immunohistochemistry as described previously. ${ }^{14,23}$ In breif, the tissue sections were washed thrice with phosphate buffered saline (PBS) and incubated with $0.3 \%$ $\mathrm{H}_{2} \mathrm{O}_{2}$ in methanol: PBS (1:1) solution for $30 \mathrm{~min}$ to quench the endogenous peroxidase activity. Slides were then washed with PBS and incubated with normal serum (Vectastain ABC kit, Vector Laboratories, Burlingame, CA, USA) for 30 min for blocking the nonspecific immunostaining. The sections were then incubated with a $1: 100$ dilution of anti-MUC1 (HMFG-2) ${ }^{24}$ or MUC4 (8G7) ${ }^{23}$ or MUC16 (OC125) ${ }^{25}$ mouse monoclonal antibodies at room temperature for $1 \mathrm{~h}$. To confirm the specificity of the IHC staining, one set of the slides was incubated with PBS without any primary antibody (no antibody control). Slides were washed $(3 \times 5 \mathrm{~min})$ with $\mathrm{PBS}$ containing $0.05 \%$ Tween-20 (PBS-T) and the sections were incubated with biotinylated- secondary antibody for $30 \mathrm{~min}$ slides were washed again $(3 \times 5 \mathrm{~min})$ with PBS-T before incubation with ABC solution (Vector Laboratories) at room temperature. The reaction color was developed by treating the tissue sections with 3, 3-diaminobenzidine (DAB) substrate (DAB substrate kit, Vector Laboratories) as per the manufacturer's instructions. A reddish-brown precipitate indicated positive immunoreactivity. The slides were washed with water, counter-stained with hematoxylin, dehydrated and mounted with Vectamount permanent mounting media (Vector Laboratories). 


\section{Assessment of Antigen Staining}

All slides were analyzed using a Nikon Eclipse E400 Microscope (Japan). The intensity of immunoreactivity of the MUC1, MUC4 and MUC16 was scored. Staining intensity was graded on a 0 to 3 scale ( 0 for no staining, + for weak immunoreactivity; ++ for moderate immunoreactivity; and +++ for strong immunoreactivity). The percentage of cells that showed positive immunoreactivity within the normal epithelial/cancerous region of the section was scored as follows: 1 for $0-25 \%$; 2 for $26-50 \%$; 3 for $51-75 \%$; and 4 for $76-100 \%$. The values of the staining intensity and the percent of immunoreactive cells were multiplied to obtain a composite score ranging from 0 to 12

\section{Statistical Analysis}

Paired $t$-tests were used to analyze the significant differences between MUC1, MUC4 and MUC16 immunostaining in all samples and also in early(low) and late- (advanced) stage subgroups. $P$-values have not been adjusted for multiple comparisons. Overall survival times were calculated as months from diagnosis to death from any cause at the date of last follow-up. Patients alive at last follow-up were treated as censored. Survival distributions were estimated using the Kaplan-Meier analysis and the log-rank test was used to compare distributions between composite staining score categories. $P$-values less than 0.05 were considered to be statistically significant.

\section{Results}

\section{MUC4 is Overexpressed in Ovarian Tumors}

An aberrant expression of MUC4 has previously been reported in ovarian tumors using Northern blot analysis and in situ hybridization. ${ }^{22,26}$ In the present study, our goal was to analyze the expression of MUC4 in situ at protein level. Owing to immunohistochemistry being more practical for routine clinical testing and a direct measure of protein level, we performed IHC profiling in normal ovary $(n=10)$ and tumor $(n=63)$ tissues utilizing a MUC4specific monoclonal antibody, 8G7. This antibody was developed in our laboratory and has been well characterized and used effectively in previous studies for similar applications. ${ }^{27,28}$ MUC4 was detected in $92 \%$ of ovarian tumor samples with a differential immunostaining $(+1 \quad(14.0 \%),+2$ $(43.0 \%)$ and $+3(35.0 \%))$, while no or very faint staining was observed in the normal ovarian epithelial cells (Table 1, Figure 1a and b). No background staining was detected in the sections incubated without the anti-MUC4 antibody confirming the specificity of the IHC assay (Figure 1c). A statistical difference $(P<0.0001)$ between the mean composite scores for MUC4 staining in the normal ovary (0.6) and tumor tissue (4.1) was observed (Table 2).

\section{Comparative Immunoprofiling of MUC1, MUC4 and MUC16 in Ovarian Carcinoma}

Our previous data showed that MUC4 was aberrantly expressed in ovarian carcinoma and could be a potential tumor marker (Figure 1). Therefore, we next compared its expression profile with MUC1 and MUC16, the two mucins overexpressed in ovarian cancer, to evaluate its potential utility as a marker for disease diagnosis. MUC16/CA125 is an established diagnostic marker for ovarian carcinoma and is being routinely used in clinics. ${ }^{29}$ IHC analyses demonstrated differential expression profiles of MUC1, MUC4 and MUC16 (Table 1). Of the total 63 ovarian tumor samples, 30.0, 46.0 and $6.0 \%$ samples showed $+1,+2$ and +3 intensity grade immunoreactivity for MUC1, respectively, with a mean composite score of $3.17 \pm 0.34$ (Table 1). For

Table 1 Expression profile and mean composite scores of MUC1, MUC4 and MC16 in ovarian tumors $(n=63)$

\begin{tabular}{|c|c|c|c|c|c|c|c|}
\hline \multirow[t]{2}{*}{ Antigen } & \multirow{2}{*}{$\begin{array}{l}\text { Staining } \\
\text { intensity }\end{array}$} & \multirow{2}{*}{$\begin{array}{l}\text { No. of } \\
\text { cases }\end{array}$} & \multirow{2}{*}{$\begin{array}{l}\text { Composite score } \\
\text { (mean } \pm \text { s.e.) }\end{array}$} & \multicolumn{3}{|c|}{ Pairwise P-value } & \multirow{2}{*}{$\begin{array}{l}\text { Overall } \\
\mathrm{P} \text {-value }\end{array}$} \\
\hline & & & & MUC1 vs MUC4 & MUC1 vs MUC16 & MUC4 vs MUC16 & \\
\hline MUC1 & $\begin{array}{c}- \\
+ \\
++ \\
+++\end{array}$ & $\begin{array}{c}11(17.5 \%) \\
19(30.2 \%) \\
29(46.0 \%) \\
4(6.3 \%)\end{array}$ & $3.17 \pm 0.34$ & & & & \\
\hline MUC4 & $\begin{array}{c}- \\
+ \\
++ \\
+++\end{array}$ & $\begin{array}{c}5(8.0 \%) \\
9(14.2 \%) \\
27(43.0 \%) \\
22(35.0 \%)\end{array}$ & $4.11 \pm 0.41$ & 0.06 & 0.58 & 0.23 & 0.029 \\
\hline MUC16 & $\begin{array}{c}- \\
+ \\
++ \\
+++\end{array}$ & $\begin{array}{l}13(20.6 \%) \\
14(22.2 \%) \\
21(33.3 \%) \\
15(23.8 \%)\end{array}$ & $3.47 \pm 0.44$ & & & & \\
\hline
\end{tabular}

$+1,+2$ and +3 indicate the intensity of immunostaining, s.e. $=$ standard error. 

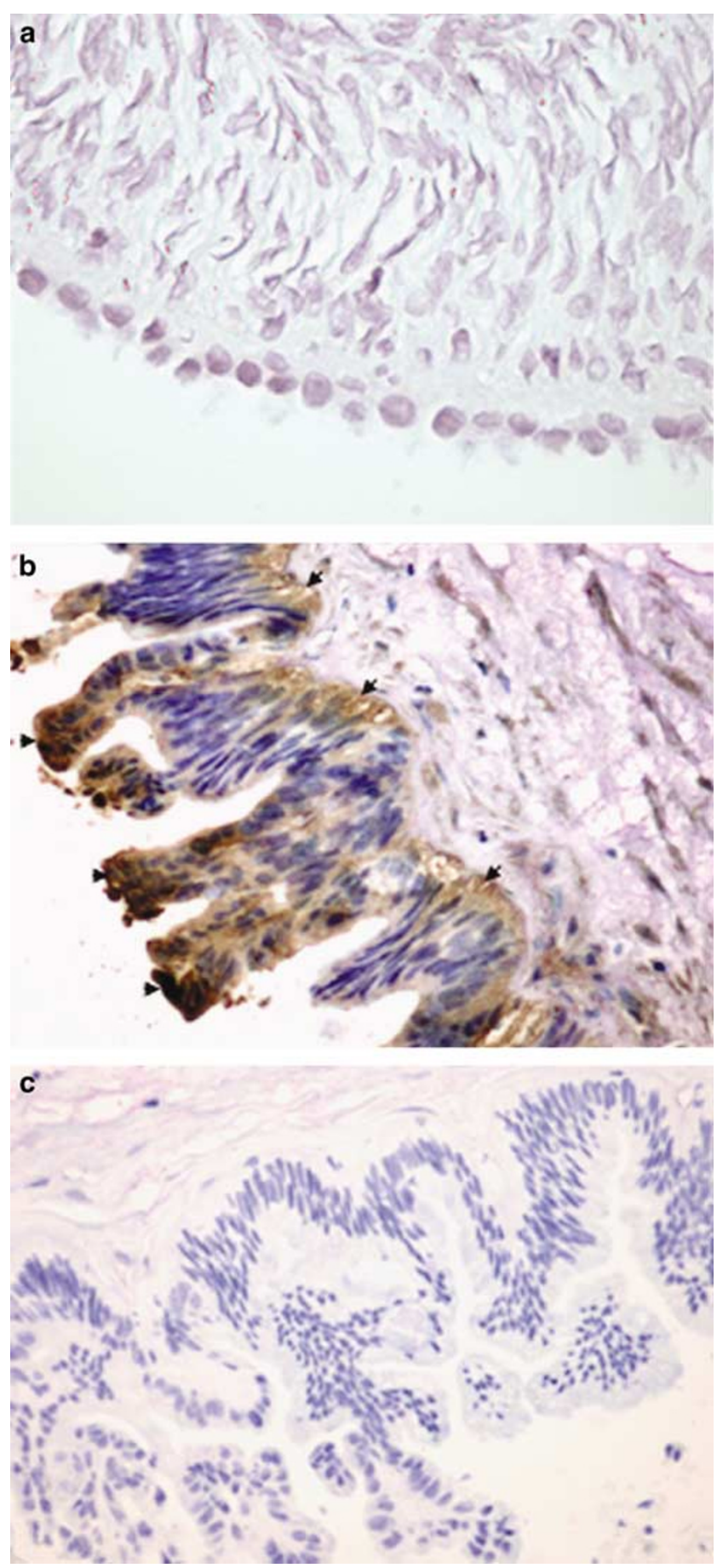

Figure 1 The expression of MUC4 in normal ovary and ovarian tumor tissues by immunohistochemistry. Tissue sections (normal and tumor ovary), cut from the paraffin-embedded blocks were probed with anti-MUC4 MAb after nonspecific blocking with serum. Anti-MUC4 antibodies showed very faint or no staining in normal ovarian epithelial cells (a) and a strong reactivity was observed in ovarian tumors (b). Note a strong immunoperoxidase staining in the apical region and a moderate staining in the basal region (arrows). No nonspecific reactivity was detected in the tissue section(s) incubated without primary antibody (c). Original magnifications $-\times 400$

MUC16, $+1,+2$ and +3 grade immunostaining was detected in $22.0,33.0$ and $23.0 \%$ of the samples, respectively, with a mean composite score of
Table 2 Comparison of the mean composite scores in normal ovarian tissues $(n=10)$ and ovarian tumor $(n=63)$ tissue samples

\begin{tabular}{lcccc}
\hline Antigen & $\begin{array}{c}\text { Mean composite } \\
\text { score for } \\
\text { staining intensity } \\
\text { (mean } \pm \text { s.d.) }\end{array}$ & $\begin{array}{c}\text { Difference } \\
\text { between } \\
\text { mean } \\
\text { composite } \\
\text { scores }\end{array}$ & P-value \\
\cline { 2 - 3 } & $\begin{array}{c}\text { Normal } \\
\text { ovary }\end{array}$ & $\begin{array}{c}\text { Ovarian } \\
\text { tumor }\end{array}$ & & \\
\hline MUC1 & $1 \pm 1.8$ & $3.2 \pm 2.7$ & 2.2 & 0.018 \\
MUC4 & $0.6 \pm 0.8$ & $4.1 \pm 3.3$ & 3.5 & $<0.0001$ \\
MUC16 & $0.5 \pm 0.7$ & $3.5 \pm 3.6$ & 3.0 & $<0.0001$ \\
\hline
\end{tabular}

$3.47 \pm 0.44$ (Table 1). No significant difference in the mean composite scores of MUC1, MUC4 and MUC16 was observed in a pairwise or overall comparison, suggesting their comparable overexpression in ovarian tumors (Table 1). In addition, all three mucin antigens displayed significant overexpression $(P<0.05)$ in ovarian tumor compared to normal ovary (Table 2 ) and the difference in mean composite score values were very highly significant $(P<0.0001)$ for both MUC4 and MUC16. Of interest, the difference between the mean composite scores of normal ovary and ovarian tumor was higher for MUC4 (3.5) compared to that of MUC16 (3.0) (Table 2). A similar immunostaining pattern of MUC1, MUC4 and MUC16 was observed in both frozen and paraffin-embedded sections.

\section{Expression Analysis of MUCs (MUC1, MUC4 and MUC16) among Different Subtumor Types}

Based on the morphological differences, we grouped the available tumor samples into four major histological types (clear cell, serous, mucinous and endometrioid). Of the 63 tumor samples, four tumor tissues (two borderline, one granulosa and one transitional type) were excluded. Remaining 59 tumor samples were assigned as clear cell $(n=9)$, serous $(n=36)$, mucinous $(n=5)$ and endometrioid $(n=9)$ subtumor types. MUC1 and MUC4 staining was mainly observed in the cytoplasm and cell membrane in majority of the cases, except for the endometrioid tumor types, which showed a clear staining in the apical and basal region of cells with a faint staining in the cytoplasm (Figure 2). On the other hand, MUC16 was specifically observed at the cell surface in the apical region in the majority of the cases, except for the clear-cell type, where it was also detected in the cytoplasm (Figure 2). The expression of MUC1, MUC4 and MUC16 was detected in all tumor types. However, among mucinous ovarian tumors, $100 \% \quad(n=5)$ of the samples were positive for MUC4, while only 40 and $20 \%$ showed immunoreactivity for MUC1 and MUC16, respectively (Figure 2). 

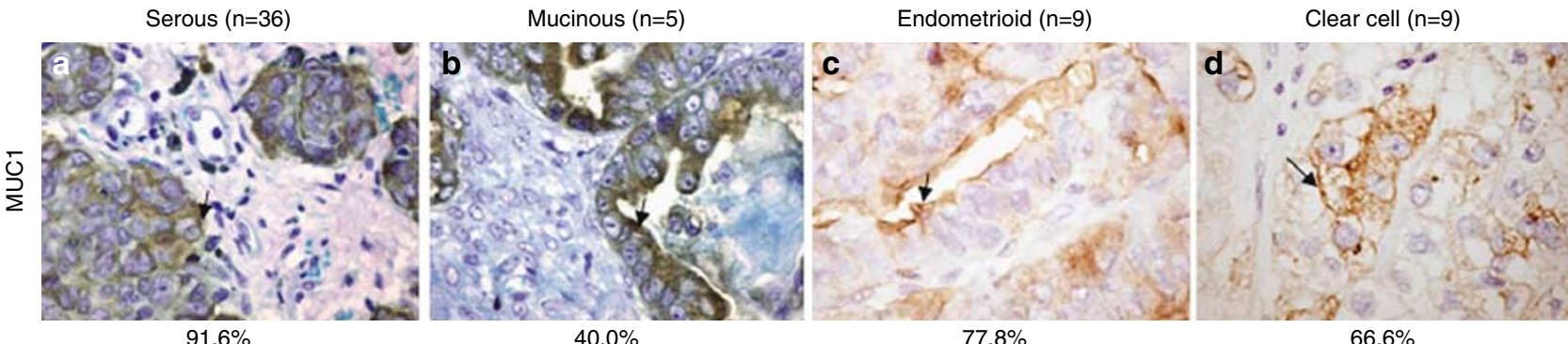

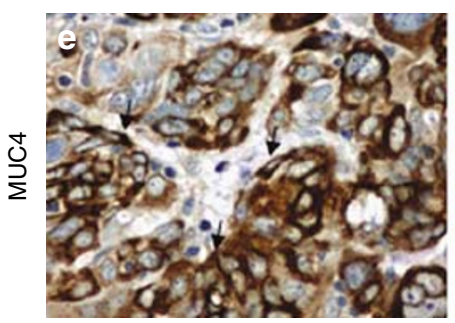

$91.6 \%$

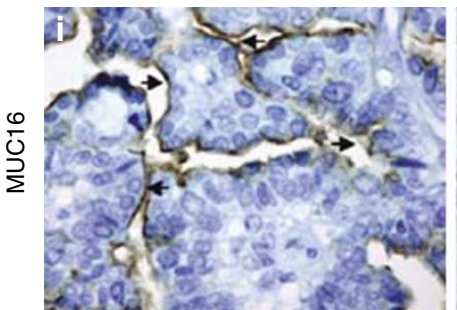

$88.9 \%$

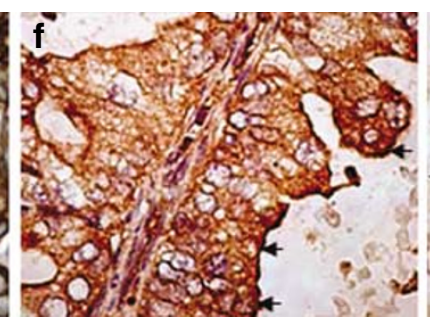

$100.0 \%$

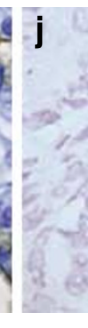

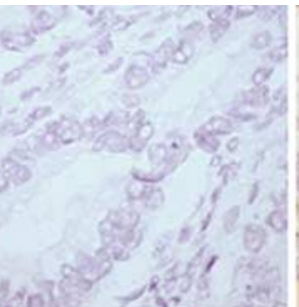

$20.0 \%$

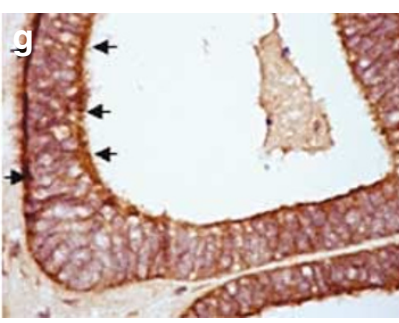

$100.0 \%$

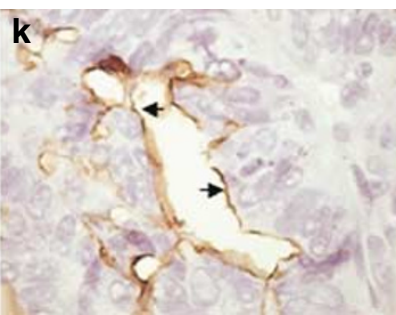

$77.8 \%$

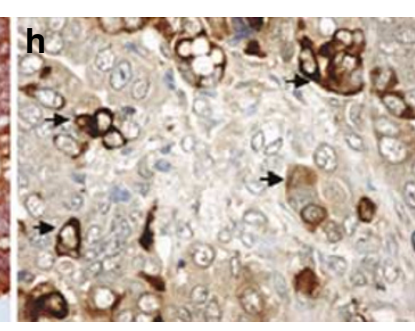

$88.8 \%$

Figure 2 The expression of MUC1, MUC4 and MUC16 in four major histological types of epithelial ovarian tumors. (a, e, i) Serous tumor; $(\mathbf{b}, \mathbf{f}, \mathbf{j})$ mucinous tumor; (c, g, k) endometrioid tumor; (d, h, l) clear cell carcinoma. Representative photographs for the expression of MUC1, MUC4 and MUC16 are shown and the percentage of positive cases for a given mucin in each histological type is indicated at the bottom in panels. Original magnifications $-\times 400$.

\section{Expression of MUC1, MUC4 and MUC16 at Different Tumor Stages}

Tumor samples were further grouped into two categories based on the clinical stage of the tumor tissue (early stage (stage I and II) and advanced stage (stage III and IV)). Of the 63 tumor tissues, 19 samples were grouped as early stage, while 43 samples were assigned as advanced stage (Table 3). One granulosa tumor type sample that did not show epithelial cell morphology was excluded. Among early-stage samples, low $(+1)$ to moderate $(+2)$ staining was observed for MUC1 and MUC16 in majority of the cases and only $5.0 \%$ of the samples showed strong $(+3)$ staining. No reactivity was observed in $21.0 \%$ of the tumor samples for MUC1 and MUC16 (Table 3). On the other hand, a positive staining for MUC4 was observed in all the cases with $+1,+2$ and +3 grade immunoreactivity in $21.0,47.0$ and $32.0 \%$ of the cases, respectively. In addition, the mean composite score value for MUC4 was significantly higher than that of MUC1 $(P=0.004)$ and MUC16 $(P=0.003)$ (Table 3$)$, indicating its greater potential for early detection of the disease. Among late-stage tumors, 84.0, 88.0 and
$81.0 \%$ of the samples showed immunoreactivity for MUC1, MUC4 and MUC16, respectively. While majority of the cases showed moderate staining for all three antigens, only a small number of samples $(5.0 \%)$ were strongly positive for MUC1. Nevertheless, 37.0 and $30.0 \%$ of the samples showed strong immunoreactivity for MUC4 and MUC16, respectively. No significant difference, however, was noted among mean composite scores of MUC1, MUC4 and MUC16 (Table 3). Altogether, MUC4 emerged as a most sensitive marker detecting 100 and $88.0 \%$ of the early and late-stage cases, respectively (Figure 3a). In addition, MUC4 in combination with either MUC1 or MUC16 detected all early and late-stage tumor samples, albeit it also resulted in the increased number of positive cases in normal ovarian tissues (Figure 3a). At a set cutoff, 2 (composite score), MUC4 still detected 95.0 and $77.0 \%$ of early and late-stage tumor cases, respectively with a considerable reduction in the number of false-positive cases (Figure 3b). The sensitivity of the detection was further improved $(95.0 \%)$ for latestage ovarian tumors, when MUC4 was used in combination with MUC16, while the number of false-positive cases remained unchanged (Figure 
Table 3 Comparison of MUC1, MUC4 and MUC16 immunostaining intensity in early $(n=19)$ and advanced stage ovarian tumors $(n=43)$

\begin{tabular}{|c|c|c|c|c|c|c|c|c|c|}
\hline & \multirow[t]{2}{*}{ Antigen } & \multicolumn{4}{|c|}{ Staining intensity (\% cases) } & \multirow{2}{*}{$\begin{array}{c}\text { Mean } \\
\text { composite } \\
\text { scores }\end{array}$} & \multirow{2}{*}{$\begin{array}{c}\text { Paired } \\
\text { antigens }\end{array}$} & \multirow{2}{*}{$\begin{array}{c}\text { Difference } \\
\text { between } \\
\text { means }\end{array}$} & \multirow[t]{2}{*}{ P-value } \\
\hline & & 0 & + & ++ & +++ & & & & \\
\hline \multirow[t]{3}{*}{ Early stage (I-II) } & MUC1 & $4(21.0 \%)$ & $7(36.8 \%)$ & $7(36.8 \%)$ & $1(5.3 \%)$ & 2.68 & MUC1-MUC4 & 2.70 & $0.004^{*}$ \\
\hline & MUC4 & $0(0.0 \%)$ & $4(21.0 \%)$ & $9(47.4 \%)$ & $6(31.6 \%)$ & 5.42 & MUC1-MUC16 & 0.16 & 0.87 \\
\hline & MUC16 & $4(21.0 \%)$ & $6(31.6 \%)$ & $8(42.1 \%)$ & $1(5.3 \%)$ & 2.84 & MUC4-MUC16 & 2.60 & $0.003^{*}$ \\
\hline \multirow[t]{3}{*}{ Late stage (III-IV) } & MUC1 & $7(16.3 \%)$ & $12(27.9 \%)$ & $22(51.2 \%)$ & $2(4.6 \%)$ & 3.38 & MUC1-MUC4 & 0.16 & 0.78 \\
\hline & MUC4 & $5(11.6 \%)$ & $4(9.3 \%)$ & $18(41.9 \%)$ & $16(37.2 \%)$ & 3.54 & MUC1-MUC16 & 0.36 & 0.58 \\
\hline & MUC16 & $8(18.6 \%)$ & $8(18.6 \%)$ & $14(32.6 \%)$ & $13(30.2 \%)$ & 3.75 & MUC4-MUC16 & 0.20 & 0.75 \\
\hline
\end{tabular}

One granulosa cell ovarian tumor sample was not included in this analysis.

${ }^{*}$ MUC4 was significantly greater than MUC1 $(P=0.004)$ and MUC16 $(P=0.003)$ in the early stage group.

$0,+,++$ and +++ indicate the intensity of immunostaining.
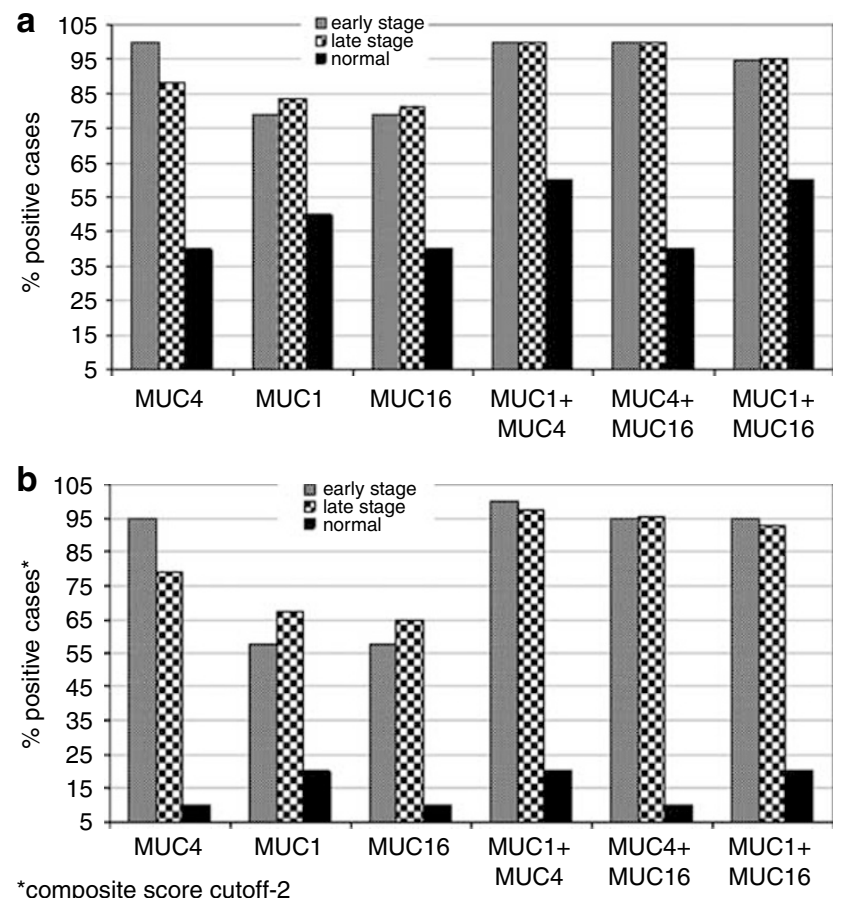

Figure 3 The sensitivity and specificity of MUC1, MUC4 and MUC16 alone and in combination with each other for the diagnosis of early- and late-stage ovarian tumor samples. (a) The percent positive cases of early and advanced-stages ovarian tumors and normal ovary are plotted for each marker. MUC4 alone shows 100 and $88.0 \%$ sensitivity for early- and advancedstage tumors, respectively. On the other hand, MUC1 and MUC16 showed only $79 \%$ and less than $84 \%$ sensitivity in early and advanced-stage samples, respectively. The specificity of MUC4 was better than MUC1 and comparable to MUC16. In addition, MUC4 in combination with MUC1 and MUC16 exhibited 100\% sensitivity even for late-stage tumors. (b) When the percent positive cases are plotted at a cutoff value 2 of composite score, MUC4 alone showed 95\% sensitivity for early-stage tumors and the highest sensitivity $(77.0 \%)$ for advanced-stage tumors. MUC4 in combination with MUC16 detected $\sim 95 \%$ of the advance-stage tumors without loosing the specificity, however, no gain in sensitivity was recorded for early-stage tumors.

3b). Therefore, our data suggest that MUC4 alone or together with MUC16 is a good marker for early- and late-stage diagnosis of ovarian carcinoma.

\section{Prognostic Significance of MUC1, MUC4 and MUC16 Expression}

Correlation between the patient's survival and the expression of MUC1, MUC4 and MUC16 was examined using Kaplan-Meier analysis. Of 38 patients with complete survival information, 23 (61\%) had died and 15 (39\%) were alive at last contact. The median follow-up for patients alive at last contact is 84 months (range: 60-108 months). For the individual MUC, staining variables $\leq 2$ or $>2$ was used as a cutoff point for staining. In the combination analysis (MUC4 + MUC16), the composite score was calculated by adding the composite score values of MUC4 and MUC16. The composite score of MUC4 + MUC16 ranged from 1 to 16 and $\leq 10$ or $>10$ was used as a cutoff point.

In our analyses, MUC1 did not show any correlation in estimated survival distributions (Figure 4a), while a trend toward poor survival was observed for MUC4 ( $P=0.079)$ (Figure 4b). Importantly, a statistically significant correlation $(P=0.046)$ for MUC16 was observed with the poor survival of the patients (Figure 4c). MUC4 and MUC16 expression in combination also showed an improved trend toward poor patient survival, although it was still insignificant $(P=0.057)$ (Figure 4d).

\section{Discussion}

Regardless of the progress made in recent years, ovarian cancer mortality has remained relatively constant for more than three decades. ${ }^{1}$ This is largely attributed to the lack of early detection, as most ovarian carcinomas $(>75 \%)$ are clinically detected at late stages, ${ }^{4}$ underscoring the need for the identification of novel biomarkers for the early detection. These biomarkers can be used alone and/ or in combination with currently available diagnostic markers/technologies to achieve greater sensitivity and a reduced false-positive rate of screening. In the present study, we evaluated the clinical significance of MUC4 mucin expression in ovarian carcinomas along with two other mucin antigens, 

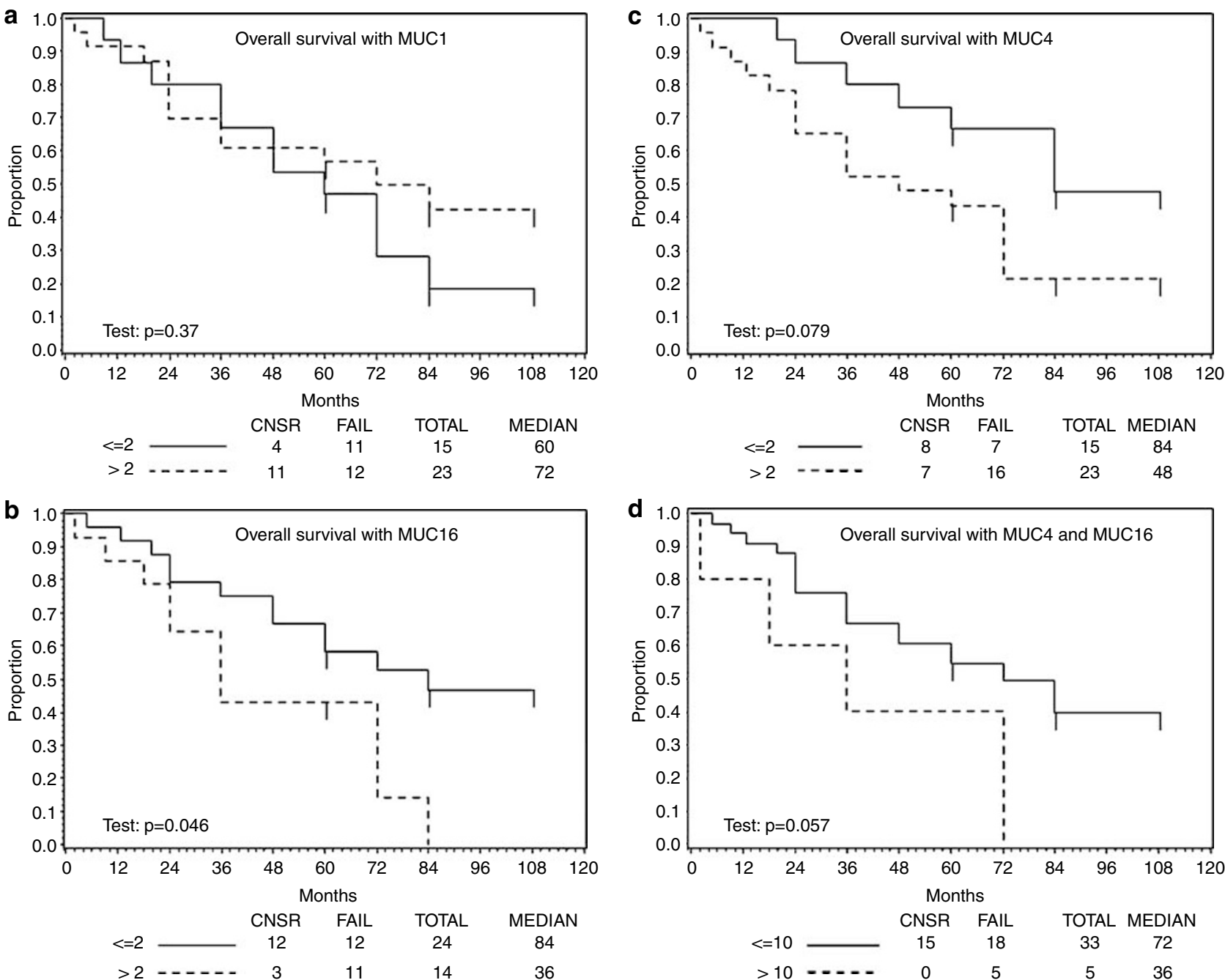

Figure 4 Kaplan-Meier analysis for the correlation of MUC1 (a), MUC4 (b), MUC16 (c) and MUC4 + MUC16 (d) expression with the overall survival of ovarian cancer patients. A significant correlation $(P=0.046)$ was observed between MUC16 expression and poor patient survival, while in cases that were positive for MUC4, a trend toward poor survival was noted $(P=0.079)$. CNSR, patient censored (alive); FAIL, patients dead; TOTAL, total number of cases; MEDIAN, median survival (in months) and composite score values of immunostaining indicated as $\leq 2$ and $>2(\mathbf{a}, \mathbf{b}, \mathbf{c}),<10$ and $>10$ (d)

MUC1 and MUC16/CA125. Our data clearly demonstrate that MUC4 is upregulated in most of the tumor tissues examined (Table 1), while no or faint staining is observed in normal ovary samples (Figure 1, Table 2). MUC1 and MUC16 also exhibited differential levels of immunoreactivity; however, the corresponding difference between mean composite scores (normal and ovarian tumor) was statistically significant $(P<0.0001)$ for MUC16 only (Table 2). In an additional analysis where we grouped the tumor samples in two categories (early and late stages), MUC4 emerged as an independent marker and detected $100 \%$ of the early-stage tumor samples (Table 3). Moreover, MUC4 exhibited the highest percentage of reactivity even among latestage tumors. The profiles of mucin gene expression in different histological types revealed the highest sensitivity of MUC4 for mucinous, endometrioid and clear-cell carcinomas; however, the sample size was limited to determine any significant correlation (Figure 2).

The pattern of mucins (MUC1, MUC4 and MUC16) expression reported herein is consistent with previous reports in ovarian cancer. ${ }^{3,18,22}$ MUC16/CA125 is the most thoroughly investigated biomarker in ovarian carcinoma and is currently in use for disease diagnosis. ${ }^{2-4}$ In earlier studies, MUC4 expression in ovarian carcinoma was also examined at the transcript level using Northern blot and in situ hybridization analyses. ${ }^{22,26}$ It was reported that MUC4 expression significantly decreased in advanced cancer stages; however, the status of MUC4 expression in the normal ovary was not studied. ${ }^{22}$ In the present report, we examined the MUC4 expression by IHC analysis in normal ovary and ovarian tumor tissues. In agreement with previous study, ${ }^{22}$ 
we also observed a downregulation of MUC4 expression in advanced-stage ovarian tumors as compared to the early-stage tumors. However, we did not see a positive correlation of MUC4 with patient's prognosis. Nonetheless, our data demonstrated a trend toward poor survival in patients with MUC4 expressing ovarian tumors (Figure 4). These contradicting observations may either be due to sample variation or due to the difference in the analytical approach. In the previous study, ${ }^{22}$ expression of MUC4 was studied at the mRNA level, which is subject to post-transcriptional regulation. In addition, as tumor is heterogeneous population of cell types, the use of Northern blot analysis utilizing tumor tissue RNA may result in reduced sensitivity. IHC analysis, on the other hand, is a powerful technique that analyzes gene expression in situ. Moreover, it can also provide information regarding the subcellular localization of the protein, which, in some cases, may also be an important determinant for clinical outcome. ${ }^{30}$

In our study, both MUC4 and MUC16 showed significant differences in the mean composite scores between normal ovary and ovarian cancer tissues, however, MUC4 displayed the highest immunoreactivity and incidence in ovarian tumors (Tables 1 and 2). The mean composite score for MUC1 in ovarian tumor tissues was comparable to that of MUC16, but no significant difference with normal ovary tissue was observed. Together, these data suggest that besides MUC16, MUC4 can also be a potential marker for ovarian cancer providing better sensitivity. Of further importance was our analyses in early- vs late-stage ovarian tumors, where MUC4 exhibited $100 \%$ sensitivity in early-stage tumors compared to 79\% sensitivity for MUC1 and MUC16 (Table 3). In a pairwise comparison, the mean composite score value for MUC4 was also significantly higher than that of MUC1 and MUC16, suggestive of its potential as a better marker for early diagnosis. Even amongst late-stage tumors, MUC4 showed highest incidence (88.0\%), however, its mean composite score value was slightly, but insignificantly, lower than that of MUC16. To depict the overall performance of all the three mucin antigens, alone and in combination, we have plotted two histograms (Figure 3a and b). The first histogram considers the entire positive cases (Figure 3a), while in the second one, we made a cutoff of 2 for composite score value to reduce the number of cases detected in the normal ovary (Figure 3b). This analysis clearly demonstrates that MUC4 alone and/or in combination with MUC16 is a good marker for the diagnosis of ovarian cancer.

In different histological types of ovarian tumors, our data demonstrated an apparent overexpression of MUC1, MUC4 and MUC16 in all tumor types except mucinous tumors (Figure 2). However, MUC4 was the only mucin that was detected in all the cases of mucinous and endometrioid tumor types. This is an important observation and needs to be explored further, as currently, only a small percentage of mucinous tumors can be detected by CA125 (present observation and a previous study ${ }^{29}$ ). Of note, the staining of MUC1 and MUC4 was observed both in the cell membrane and in the cytoplasm in majority of the ovarian tumor samples, whereas MUC16 was predominantly localized on the cell membrane with a few exceptions of cytoplasmic staining (Figure 2). In a previous study, the high cytoplasmic staining of MUC1 has been associated with poor prognosis in breast carcinomas. ${ }^{30} \mathrm{~A}$ similar correlation could also be made for ovarian carcinoma; however, it needs to be examined in larger sets of tumor samples. In an attempt to predict the prognostic value of mucin antigens, we observed that a higher expression MUC16 is significantly correlated with the overall poor survival of ovarian cancer patients. The expression of MUC4 also indicated a trend towards poor prognosis; however, it needs to be examined further to test significance (Figure 4). These observations warrant future investigations to understand the potential involvement of these mucins in the disease pathology.

In summary, the results from this study demonstrated that MUC4 is upregulated in ovarian cancer with a relatively higher incidence in early-stage tumors. We propose that MUC4 can serve as an independent marker for early diagnosis of ovarian carcinoma and can be utilized in combination with MUC16/CA125 to achieve greater sensitivity for the detection of late-stage tumors. MUC16 alone stood as a good prognostic marker, while MUC4 showed a trend toward poor survival of the cancer patients. Future studies are needed to exploit these findings and to develop a MUC4-based analytical procedure for the early detection of ovarian carcinoma. An intraperitoneal/ascitic fluid-based assay will be more appropriate in consideration of the widespread intraperitoneal metastasis of ovarian carcinoma.

\section{Acknowledgements}

We would like to acknowledge the grant support by an Idea Award Grant from the United States Department of Defense (OC040110). In addition, we thank Erik Moore for excellent technical support and Ms Kristi LW Berger for editing the manuscript. Authors thankfully acknowledge Dr Gendler, Mayo Clinics, AZ and Drs Metzgar and Bast, Duke University Medical Center, for providing antiMUC1 and anti-MUC16 antibodies, respectively. We also thank the Director of the Tissue Bank at UNMC, Dr Julia Bridge, for providing tissue samples. The NCI Cancer Center Grant (P30 CA36727) to UNMC and CHTN Western Division, Case Western Reserve University, Cleveland, $\mathrm{OH}$, is greatly acknowledged for providing the additional tissues for this study. 


\section{References}

1 Jemal A, Murray T, Ward E, et al. Cancer statistics, 2005. CA Cancer J Clin 2005;55:10-30.

2 Fritsche HA, Bast RC. CA 125 in ovarian cancer: advances and controversy. Clin Chem 1998;44: 1379-1380.

3 Jacobs IJ, Oram DH, Bast Jr RC. Strategies for improving the specificity of screening for ovarian cancer with tumor-associated antigens CA 125, CA 15-3, and TAG 72.3. Obstet Gynecol 1992;80:396-399.

4 Menon U, Jacobs I. Screening for ovarian cancer. Best Pract Res Clin Obstet Gynaecol 2002;16:469-482.

5 Jacobs IJ, Menon U. Progress and challenges in screening for early detection of ovarian cancer. Mol Cell Proteomics 2004;3:355-366.

6 van Jr NJ, DePriest PD, Reedy MB, et al. The efficacy of transvaginal sonographic screening in asymptomatic women at risk for ovarian cancer. Gynecol Oncol 2000;77:350-356.

7 Stelmachow J, Spiewankiewicz B. Possibilities and limitation of endoscopic procedures in oncological gynaecology. Eur J Gynaecol Oncol 2005;26:21-23.

8 Kawamoto S, Urban BA, Fishman EK. CT of epithelial ovarian tumors. Radiographics 1999;263:85-102.

9 Auersperg N, Wong AS, Choi KC, et al. Ovarian surface epithelium: biology, endocrinology, and pathology. Endocr Rev 2001;22:255-288.

10 Gendler SJ, Spicer AP. Epithelial mucin genes. Annu Rev Physiol 1995;57:607-634.

11 Hollingsworth MA, Swanson BJ. Mucins in cancer: protection and control of the cell surface. Nat Rev Cancer 2004;4:45-60.

12 Moniaux N, Escande F, Porchet N, et al. Structural organization and classification of the human mucin genes. Front Biosci 2001;6:1192-1206.

13 Andrianifahanana M, Moniaux N, Schmied BM, et al. Mucin (MUC) gene expression in human pancreatic adenocarcinoma and chronic pancreatitis: a potential role of MUC4 as a tumor marker of diagnostic significance. Clin Cancer Res 2001;7: 4033-4040.

14 Singh AP, Moniaux N, Chauhan SC, et al. Inhibition of MUC4 expression suppresses pancreatic tumor cell growth and metastasis. Cancer Res 2004;64: 622-630.

15 Moniaux N, Andrianifahanana M, Brand RE, et al. Multiple roles of mucins in pancreatic cancer, a lethal and challenging malignancy. $\mathrm{Br} \mathrm{J}$ Cancer 2004;91:1633-1638.

16 Yin BW, Dnistrian A, Lloyd KO. Ovarian cancer antigen CA125 is encoded by the MUC16 mucin gene. Int J Cancer 2002;98:737-740.
17 Yin BW, Lloyd KO. Molecular cloning of the CA125 ovarian cancer antigen: identification as a new mucin, MUC16. J Biol Chem 2001;276:27371-27375.

18 Dong Y, Walsh MD, Cummings MC, et al. Expression of MUC1 and MUC2 mucins in epithelial ovarian tumours. J Pathol 1997;183:311-317.

19 Hamann PR, Hinman LM, Beyer CF, et al. A calicheamicin conjugate with a fully humanized anti-MUC1 antibody shows potent antitumor effects in breast and ovarian tumor xenografts. Bioconjug Chem 2005;16: 354-360.

20 Kong B, Wang W, Liu C, et al. Efficacy of lentivirusmediated and MUC1 antibody-targeted VP22-TK/GCV suicide gene therapy for ovarian cancer. In Vivo 2003;17:153-156.

21 Carraway KL, Price-Schiavi SA, Komatsu M, et al. Muc4/sialomucin complex in the mammary gland and breast cancer. J Mammary Gland Biol Neoplasia 2001;6:323-337.

22 Giuntoli RL, Rodriguez GC, Whitaker RS, et al. Mucin gene expression in ovarian cancers. Cancer Res 1998;58:5546-5550.

23 Moniaux N, Varshney GC, Chauhan SC, et al. Generation and characterization of anti-MUC4 monoclonal antibodies reactive with normal and cancer cells in humans. J Histochem Cytochem 2004;52:253-261.

24 Burchell J, Gendler S, Taylor-Papadimitriou J, et al. Development and characterization of breast cancer reactive monoclonal antibodies directed to the core protein of the human milk mucin. Cancer Res 1987; 47:5476-5482.

25 Bast Jr RC, Feeney M, Lazarus H, et al. Reactivity of a monoclonal antibody with human ovarian carcinoma. J Clin Invest 1981;68:1331-1337.

26 Boman F, Buisine MP, Wacrenier A, et al. Mucin gene transcripts in benign and borderline mucinous tumours of the ovary: an in situ hybridization study. J Pathol 2001;193:339-344.

27 Park HU, Kim JW, Kim GE, et al. Aberrant expression of MUC3 and MUC4 membrane-associated mucins and sialyl Le(x) antigen in pancreatic intraepithelial neoplasia. Pancreas 2003;26:48-54.

28 Swartz MJ, Batra SK, Varshney GC, et al. MUC4 expression increases progressively in pancreatic intraepithelial neoplasia. Am J Clin Pathol 2002;117: 791-796.

29 Bast Jr RC, Xu FJ, Yu YH, et al. CA 125: the past and the future. Int J Biol Markers 1998;13:179-187.

30 Rahn JJ, Dabbagh L, Pasdar M, et al. The importance of MUC1 cellular localization in patients with breast carcinoma: an immunohistologic study of 71 patients and review of the literature. Cancer 2001;91:19731982. 